\title{
Production of grazed tropical grasses in different agroecosystems in Puerto Rico. III. Semiarid'
}

\author{
Luis E. Tergas, Jaime Vélez-Santiago \\ and Angel V. Méndez-Cruz ${ }^{2}$
}

\begin{abstract}
A field experiment was conducted at the Lajas Experiment Substation in the semiarid region of Puerto Rico to evaluate the production and persistence of 8 tropical grasses, Cynodon nlemfuensis Star, C. dactylon Coastcross-1, C. plectostachyus Star, Panicum maximum USDA PI 291047, Makueni, Guinea and $P$. maximum USDA PI 259553, and the naturalized pasture Dichanthium annulatus pajon. Small plots were grazed at 3- to 5-week intervals for 2 years. P. maximum USDA PI 259553 and Makueni were highly productive in the semiarid,region, as well as in previous experiments in humid regions in Puerto Rico. They are highly recommended for grazing trials, P. maximum Makueni and USDA PI 291047 were the most productive during the first year, although they were not significantly different $(P=0.05)$ from $P$. maximum USDA PI 259553 and Common Guinea, and Cynodon plecfyostachyus Star. The production of $C$. nlemfuensis Star was similar to that of Dichanthium annulatus but higher than that of $C$. dactylon Coasterass-1 $(P=0.05)$. The average production for all grasses declined at the beginning of the year, during the cool short days in February and March, 1983, without any significant difference $(P=0.05)$ among species and cultivars. Production increased at the beginning of the rainy season, but it was low at the peak of the rainy season because of trampling in poorly aerated and compact soils. C. plectostachyus Star was the most productive grass during the second year of experiment, although it was not significantly different $(P=0.05)$ from $P$. maximum USDA PI 291017 and 259553 and cultivar Makueni. The production of Common Guinea and that of $D$. annulatus were similar and higher than that of $C$. nlemfuensis Star and $C$. dactylon Coasteross-I $(P=0.05)$, which did not persist at the end of the experiment. Forage production during the second year was lower than in the first year because of less rainfall in 1983 . The mean CP content of all grasses varied from 11.29 to 14.05, except that of D. annulatus, which was only $9.73 \%$. CP content was lower during the periods of maximum forage: production.
\end{abstract}

'Manuscript submitted to Editorial Board 29 May 1986.

${ }^{2}$ Associate Professor, Agronomy and Soils Department; Associate Agronomist (ret.), Corozal Substation; and Associate Animal Husbandman, Lajas Substation, respectively. Agricultural Experiment Station, Mayagüez Campus, University of Puerto Rico, Rio Piedras, P. R. 


\section{INTRODUCTION}

The grasslands of the southern coastal plains and adjacent foothills are characterized by almost complete absence of trees and the presence of a few scattered shrubs and forbs (2). The soils are fertile and good for agricultural use. However, the region is probably the least developed part of the island because of low rainfall and lack of water for irrigation.

Most of the work on selection of improved forage species has been done in the humid coastal northern plains and mountain regions of Puerto Rico $(6,10)$. The performance of several grasses under clipping was evaluated in the Lajas Valley, which is representative of the semiarid region of the southern coast (9). Cenchrus ciliaris Buffel USDA PI 263509 and Panicum maximum Makueni were the most productive grasses during the short cool days from November through March. $P$. maximum USDA PI 291047, Makueni and USDA PI 284765 produced similar dry forage yields, which were higher than those of the other grasses during the rest of the year when temperatures were warmer (9).

Cynodon nlemfuensis Star, C. dactylon Coastcross- 1 and C. plectostachyus Star are highly productive grasses under cutting in the humid mountain and coastal northern regions $(6,10)$, but they have not been evaluated in the southern region. Under grazing conditions in small plots in the humid regions, $C$. plectostachyus $\operatorname{Star}(7,8)$ has been more persistent than the other grasses. Further evaluation is needed to assess production in drier environments.

Dichanthium annulatus (pajón) is a naturalized grass that has invaded the southern coast, and it is the dominant species in the region. It is drought tolerant and grows well in poorly aerated and compact soils but its nutritive value is poor (2).

The objective of the present investigation was to evaluate the production and persistence of selected grass species adapted to the semiarid southern coastal region of Puerto Rico subjected to grazing in small plots.

\section{MATERIALS AND METHODS}

The experiment was conducted at the Lajas Agricultural Experiment Substation, lat. $18^{\circ} 03^{\prime} \mathrm{N}$ and long. $67^{\circ} 03^{\prime} \mathrm{W}$, at an elevation of $27 \mathrm{~m}$, in the semiarid region of Puerto Rico. Mean annual rainfall is $1102 \mathrm{~mm}$, distributed from May through October, and mean daily temperature is $19^{\circ} \mathrm{C}$, with seasonal variations of about $3^{\circ} \mathrm{C}$.

The soil is a Cartagena clay (Udic Chromusterts) with $\mathrm{pH} 6.3$ and 2 $\mathrm{p} / \mathrm{m}$ available $\mathrm{P}$ (Bray 1 ) and $132 \mathrm{p} / \mathrm{m}$ exchangeable $\mathrm{K}$ in the top $15 \mathrm{~cm}$.

A randomized block design with four replications and 8 grass cultivars (table 1) in $8 \times 8 \mathrm{~m}$ plots was established August 1981. These grasses were 
TABLE 1.-Identification of grass cultivars

\begin{tabular}{|c|c|c|c|}
\hline $\begin{array}{l}\text { Grass } \\
\text { number }\end{array}$ & Species & USDA PI ${ }^{1}$ & $\mathrm{PR}_{\mathrm{PI}^{2}}$ \\
\hline$\overline{1}$ & $\begin{array}{l}\text { Cynodon nlemfuensis var. } \\
\text { nlemfuensis cv. Star }\end{array}$ & & 2341 \\
\hline 1 & C. dactylon ev. Coasteross-1 & 255455 & 11504 \\
\hline 3 & C. plectostachyus cv. Star & 341818 & 11487 \\
\hline 4 & Panicum maximum & 291047 & 13093 \\
\hline 5 & P. maximum cv. Makueni & 349676 & 12917 \\
\hline 6 & P. maximum cv. Common Guinea & - & - \\
\hline 7 & P. maximum & 259553 & 3622 \\
\hline 8 & Dichanthium annulatus & - & 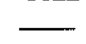 \\
\hline
\end{tabular}

fertilized with a $15-5-10$ fertilizer at a rate of $1,600 \mathrm{~kg} / \mathrm{ha} /$ year divided in 4 equal applications throughout the year. The plots were irrigated as needed.

All plots were cut at a height of $15 \mathrm{~cm}$ December 1981, and grazing started 1 month later and continued at 3- to 5-week intervals to a height of $15 \mathrm{~cm}$ with a group of 35 adult animals for a period of 1 to 2 days.

The forage offered in each plot was determined by sampling before and after grazing, following the pair quadrats method described by Rivera and Rodríguez (3). Green forage was weighed in the field and samples were taken and dried at $55^{\circ} \mathrm{C}$ in a forced air oven for $48 \mathrm{~h}$ to determine dry matter content. Monthly hand-plucked forage samples were composited by replicates of each grass treatment, dried at $55^{\circ} \mathrm{C}$ in a forced air oven, ground in a Wiley mill to pass through a $1-\mathrm{mm}$ screen and analyzed for $\mathrm{N}$ content with a Technicon Autoanalyzer. Crude protein (CP) was calculated as $\mathrm{N} \times 6.25$. The procedure was repeated over 2 consecutive years; information was for 13 grazing dates for each year.

\section{RESULTS AND DISCUSSION}

The most productive grasses during the first year of experiment from January 1982 to January 1983 were $P$. maximum Makueni and USDA PI 291047, although they were not significantly different $(\mathrm{P}=0.05)$ from P. maximum USDA PI 259553 and Common Guinea, and Cynodon plectyostachyus Star (table 2). The production of $C$. nlemfuensis Star was similar to that of Dichanthium annulatus but higher than that of $C$. dactylon Coastcross-1 $(\mathrm{P}=0.05)$. The average production for all grasses declined at the beginning of the year during the cool short days in February and March 1983 , without any significant difference $(\mathrm{P}=0.05)$ among 
TABLE 2.-Mean dry forage on offer of tropical grasses grazed in the semiarid region at Lajas, Puerto Rico, 1982-1982

\begin{tabular}{|c|c|c|c|c|c|c|c|c|c|c|c|c|c|c|c|}
\hline \multirow[b]{2}{*}{$\begin{array}{l}\text { Grass } \\
\text { number }\end{array}$} & \multicolumn{13}{|c|}{ Grazing dates } & \multirow[b]{2}{*}{$\begin{array}{c}\text { Mean per } \\
\text { grazing }\end{array}$} & \multirow[b]{2}{*}{$\begin{array}{c}\text { Total } \\
\text { per year }\end{array}$} \\
\hline & $\begin{array}{c}\operatorname{Jan}_{1982} 20 \\
\end{array}$ & $\begin{array}{c}\text { Feb. } 24 \\
1982\end{array}$ & $\begin{array}{c}\text { Apr. } 1 \\
1982\end{array}$ & $\begin{array}{c}\text { May 3 } \\
1982\end{array}$ & $\begin{array}{c}\text { May } 26 \\
1982\end{array}$ & $\begin{array}{c}\text { Jun. } 24 \\
1982\end{array}$ & $\begin{array}{c}\text { Jul. } 21 \\
1982\end{array}$ & $\begin{array}{c}\text { Aug. } 19 \\
1982\end{array}$ & $\begin{array}{c}\text { Sep. } 29 \\
1982\end{array}$ & $\begin{array}{c}\text { Oct. } 28 \\
1982\end{array}$ & $\begin{array}{c}\text { Nov. } 29 \\
1982\end{array}$ & $\begin{array}{c}\text { Jan. } 4 \\
1983\end{array}$ & $\begin{array}{c}\operatorname{Jan.31} \\
1983\end{array}$ & & \\
\hline \multicolumn{16}{|c|}{ metric ton/ha } \\
\hline 7 & $2.68 \mathrm{a}^{\mathrm{I}}$ & $2.37 \mathrm{a}$ & $0.89 \mathrm{a}$ & $1.27 \mathrm{ab}$ & $1.61 \mathrm{abc}$ & $1.37 \mathrm{a}$ & $2.08 \mathrm{ab}$ & $1.17 \mathrm{ab}$ & $1.09 \mathrm{bcd}$ & $0.90 \mathrm{~b}$ & $1.36 \mathrm{~b}$ & $1.30 \mathrm{~b}$ & $1.41 \mathrm{a}$ & $1.50 \mathrm{ab}$ & $19.48 \mathrm{ab}$ \\
\hline 4 & $2.65 \mathrm{a}$ & $1.52 \mathrm{ab}$ & $0.62 \mathrm{a}$ & $0.66 \mathrm{~b}$ & $1.70 \mathrm{ab}$ & $1.11 \mathrm{a}$ & $2.17 \mathrm{a}$ & $1.73 \mathrm{ab}$ & $2.65 \mathrm{a}$ & $0.83 b c$ & $2.66 \mathrm{a}$ & $2.21 \mathrm{a}$ & $2.17 \mathrm{a}$ & $1.75 \mathrm{a}$ & $22.68 \mathrm{a}$ \\
\hline 3 & $2.32 \mathrm{a}$ & $1.95 \mathrm{ab}$ & $0.99 \mathrm{a}$ & $1.46 \mathrm{ab}$ & $1.22 b c$ & $1.41 \mathrm{a}$ & $2.21 \mathrm{a}$ & $1.84 \mathrm{ab}$ & $0.38 \mathrm{~d}$ & $0.42 \mathrm{~cd}$ & $1.06 \mathrm{~b}$ & $1.76 \mathrm{a}$ & $1.54 \mathrm{a}$ & $1.43 \mathrm{ab}$ & $18.59 \mathrm{ab}$ \\
\hline 6 & $2.02 \mathrm{ab}$ & $1.21 \mathrm{~b}$ & $0.63 \mathrm{a}$ & $0.81 \mathrm{~b}$ & $1.18 \mathrm{bc}$ & $1.09 \mathrm{a}$ & $2.28 \mathrm{a}$ & $2.15 \mathrm{a}$ & $2.42 \mathrm{a}$ & $0.72 \mathrm{bed}$ & $2.73 a$ & $1.70 \mathrm{a}$ & $1.55 \mathrm{a}$ & $1.58 \mathrm{ab}$ & $20.48 \mathrm{ab}$ \\
\hline 5 & $1.90 \mathrm{ab}$ & $1.62 \mathrm{ab}$ & $0.38 \mathrm{a}$ & $0.65 \mathrm{~b}$ & $2.11 \mathrm{a}$ & $1.21 \mathrm{a}$ & $2.19 \mathrm{a}$ & $1.80 \mathrm{ab}$ & $2.67 \mathrm{a}$ & $1.40 \mathrm{a}$ & $2.73 \mathrm{a}$ & $3.23 \mathrm{a}$ & $1.47 \mathrm{a}$ & $1.80 \mathrm{a}$ & $23.36 \mathrm{ab}$ \\
\hline 2 & $1.88 \mathrm{ab}$ & $1.74 \mathrm{ab}$ & $0.86 \mathrm{a}$ & $1.70 \mathrm{a}$ & $0.81 \mathrm{c}$ & $1.02 \mathrm{a}$ & $1.37 \mathrm{bc}$ & $0.85 \mathrm{~b}$ & $0.52 \mathrm{~cd}$ & $0.52 \mathrm{bed}$ & $1.03 \mathrm{~b}$ & $1.52 \mathrm{a}$ & $1.38 \mathrm{a}$ & $1.17 \mathrm{e}$ & $15.22 \mathrm{c}$ \\
\hline 1 & $1.65 \mathrm{ab}$ & $1.43 \mathrm{ab}$ & $=0.99 \mathrm{a}$ & $1.33 \mathrm{ab}$ & $0.82 \mathrm{c}$ & $1.00 \mathrm{a}$ & $1.12 \mathrm{c}$ & $0.95 \mathrm{a}$ & 1.18 be & $0.34 \mathrm{~d}$ & $1.23 \mathrm{~b}$ & $3.36 \mathrm{a}$ & $1.65 \mathrm{a}$ & $1.31 \mathrm{~b}$ & $17.04 \mathrm{~b}$ \\
\hline 8 & $1.01 \mathrm{~b}$ & $1.21 \mathrm{~b}$ & $0.46 \mathrm{a}$ & $1.09 \mathrm{ab}$ & $1.26 \mathrm{bc}$ & $1.00 \mathrm{a}$ & $1.82 \mathrm{abc}$ & $1.74 \mathrm{ab}$ & $1.31 \mathrm{~b}$ & $0.50 \mathrm{bed}$ & $1.44 \mathrm{~b}$ & $2.77 \mathrm{a}$ & $1.36 \mathrm{a}$ & $1.31 \mathrm{~b}$ & $16.98 \mathrm{~b}$ \\
\hline Mean & 2.02 & 1.63 & 0.73 & 1.12 & 1.34 & 1.15 & 1.90 & 1.53 & 1.31 & 0.71 & 1.78 & 2.23 & 1.57 & 1.48 & 19.23 \\
\hline
\end{tabular}

'Means in the same column followed by the same letter do not differ statistically at the $5 \%$ probability level. 
TABLE 3.-Monthly rainfall at Lajas Substation during the course of the experiment, 1981-1983

\begin{tabular}{lrrr}
\hline Month & 1981 & 1982 & 1983 \\
\hline & & $m m$ & \\
January & 10 & 13 & 15 \\
February & 35 & 30 & 18 \\
March & 50 & 7 & 121 \\
April & 98 & 42 & 105 \\
May & 129 & 155 & 243 \\
June & 90 & 43 & 16 \\
July & 52 & 128 & 31 \\
August & 103 & 190 & 133 \\
September & 164 & 211 & 24 \\
October & 168 & 135 & 152 \\
November & 71 & 137 & 160 \\
December & 175 & 55 & 76 \\
Total & 1,145 & 1,146 & 1,093 \\
\hline
\end{tabular}

species and cultivars; then production increased at the beginning of the rainy season in May (table 3), but it was low at the peak of the rainy season in October (fig. 1), probably because of trampling in poorly aerated and compact soils.

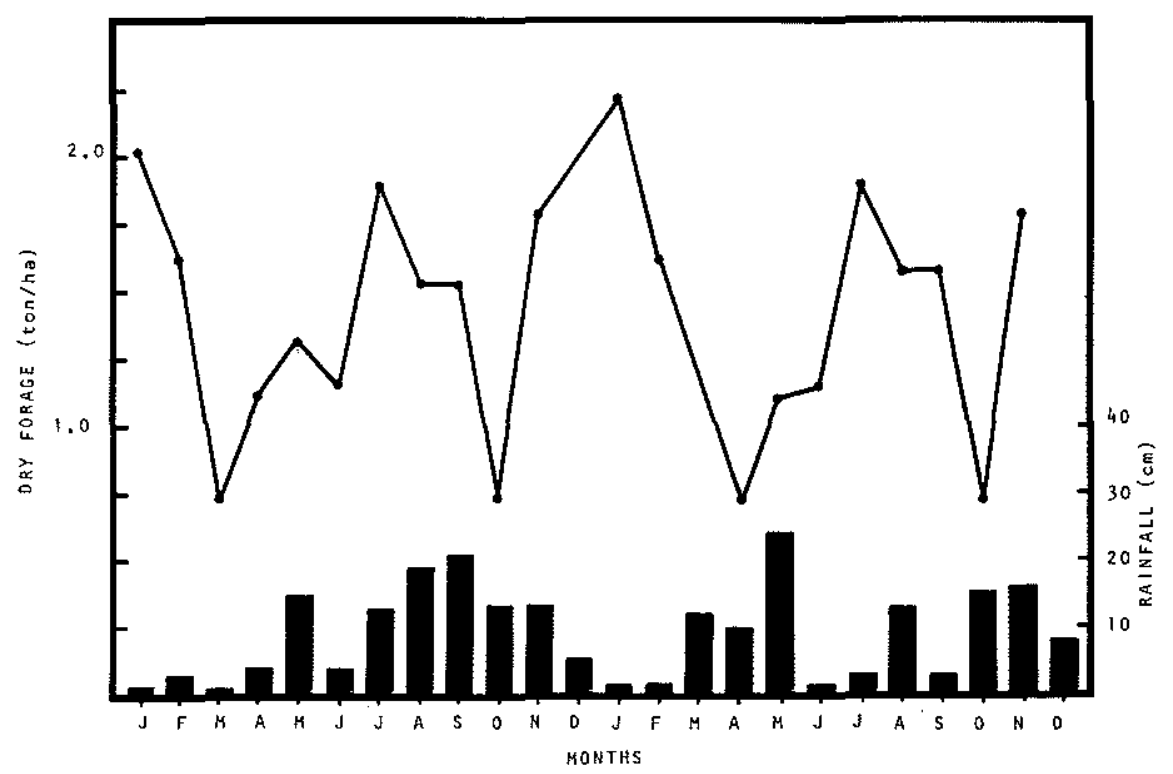

FIG. 1. Seasonal dry forage on offer, mean for all grasses, grazed in small plots every 3 to 5 weeks at Lajas, Puerto Rico, 1982 to 1984. 
TABLE 4.-Mean dry forage on offer of tropical grasses grazed in the semiarid region at Lajas, Puerto Rico, 1983-1984

\begin{tabular}{|c|c|c|c|c|c|c|c|c|c|c|c|c|c|c|c|}
\hline \multirow[b]{2}{*}{$\begin{array}{l}\text { Grass } \\
\text { number }\end{array}$} & \multicolumn{13}{|c|}{ Grazing dates } & \multirow[b]{2}{*}{$\begin{array}{l}\text { Mean per } \\
\text { grazing }\end{array}$} & \multirow[b]{2}{*}{$\begin{array}{c}\text { Total } \\
\text { per year }\end{array}$} \\
\hline & $\begin{array}{c}\text { Feb. } 28 \\
1983\end{array}$ & $\begin{array}{c}\text { Mar. 25 } \\
1983\end{array}$ & $\begin{array}{c}\text { Apr. } 22 \\
1983\end{array}$ & $\begin{array}{c}\text { May } 17 \\
1983\end{array}$ & $\begin{array}{c}\text { Jun. } 6 \\
1983\end{array}$ & $\begin{array}{c}\text { Aug. } 5 \\
1983\end{array}$ & $\begin{array}{l}\text { Sep. I } \\
1983\end{array}$ & $\begin{array}{c}\text { Sep. } 23 \\
1983\end{array}$ & $\begin{array}{l}\text { Oct. } 21 \\
1983\end{array}$ & $\begin{array}{c}\text { Nov. } 14 \\
1983\end{array}$ & $\begin{array}{c}\text { Dec. } 7 \\
1983\end{array}$ & $\begin{array}{c}\text { Jan. } 12 \\
1983\end{array}$ & $\begin{array}{c}\text { Feb. } 17 \\
1984\end{array}$ & & \\
\hline \multicolumn{16}{|c|}{ metric tons/ ha } \\
\hline 3 & $0.87 \mathrm{a}^{1}$ & $1.88 \mathrm{a}$ & $1.65 \mathrm{a}$ & $1.16 \mathrm{ab}$ & $0.99 \mathrm{ab}$ & $2.02 \mathrm{a}$ & $0.40 \mathrm{ed}$ & $1.62 \mathrm{a}$ & $1.80 \mathrm{abc}$ & $1.66 \mathrm{a}$ & $1.20 \mathrm{a}$ & $1.66 \mathrm{a}$ & $1.30 \mathrm{a}$ & $1.40 \mathrm{a}$ & $18.21 \mathrm{a}$ \\
\hline 8 & $0.82 \mathrm{a}$ & $1.26 \mathrm{ab}$ & $0.89 a$ & $1.12 \mathrm{ab}$ & $0.59 \mathrm{~b}$ & $1.69 \mathrm{ab}$ & $0.51 \mathrm{~cd}$ & $1.88 \mathrm{a}$ & $1.08 \mathrm{bc}$ & $0.61 \mathrm{~b}$ & $0.82 \mathrm{ab}$ & $0.66 \mathrm{bc}$ & $0.55 \mathrm{bc}$ & $0.96 \mathrm{c}$ & $12.50 \mathrm{c}$ \\
\hline 2 & $0.80 \mathrm{a}$ & $1.29 \mathrm{ab}$ & $1.15 \mathrm{a}$ & $0.65 \mathrm{~b}$ & $0.82 \mathrm{ab}$ & $0.72 \mathrm{e}$ & $0.48 \mathrm{ed}$ & $1.01 \mathrm{a}$ & $1.23 \mathrm{abc}$ & $0.43 \mathrm{~b}$ & $0.00 \mathrm{c}$ & $0.00 \mathrm{e}$ & $0.00 \mathrm{~d}$ & $0.66 \mathrm{~d}$ & $8.58 \mathrm{~d}$ \\
\hline 4 & $0.73 \mathrm{a}$ & $0.97 \mathrm{~b}$ & $1.53 \mathrm{a}$ & $1.27 \mathrm{ab}$ & $1.26 \mathrm{a}$ & $1.04 \mathrm{bc}$ & $1.06 \mathrm{bc}$ & $0.93 \mathrm{a}$ & $2.07 \mathrm{a}$ & $2.32 \mathrm{a}$ & $1.12 \mathrm{a}$ & $0.65 \mathrm{bc}$ & $1.80 \mathrm{ab}$ & $1.29 \mathrm{ab}$ & $16.75 \mathrm{ab}$ \\
\hline 7 & $0.72 \mathrm{a}$ & $1.15 \mathrm{ab}$ & $1.15 \mathrm{a}$ & $1.54 \mathrm{ab}$ & $0.09 a b$ & $1.28 \mathrm{abc}$ & $1.82 \mathrm{a}$ & $2.02 \mathrm{a}$ & $1.11 \mathrm{bc}$ & $2.11 \mathrm{a}$ & $1.59 \mathrm{a}$ & $1.05 \mathrm{~b}$ & $1.21 \mathrm{abc}$ & $1.36 \mathrm{ab}$ & $17.63 \mathrm{ab}$ \\
\hline 6 & $0.53 \mathrm{a}$ & $1.09 \mathrm{ab}$ & $1.30 \mathrm{a}$ & $1.02 \mathrm{ab}$ & $1.13 \mathrm{ab}$ & $0.54 \mathrm{c}$ & $1.05 \mathrm{bc}$ & $0.74 \mathrm{a}$ & $1.93 \mathrm{ab}$ & $2.17 \mathrm{a}$ & $0.74 \mathrm{ab}$ & $0.51 \mathrm{bed}$ & $2.32 \mathrm{a}$ & $1.16 \mathrm{be}$ & $15.05 \mathrm{bc}$ \\
\hline 1 & $0.50 \mathrm{a}$ & $1.34 \mathrm{ab}$ & $1.35 \mathrm{a}$ & $1.02 \mathrm{ab}$ & $0.60 \mathrm{~b}$ & $0.80 \mathrm{bc}$ & $0.05 \mathrm{~d}$ & $0.59 \mathrm{a}$ & $0.88 \mathrm{c}$ & $0.70 \mathrm{~b}$ & $0.59 \mathrm{ab}$ & $0.33 \mathrm{~cd}$ & $0.34 \mathrm{c}$ & $0.70 \mathrm{~d}$ & $9.09 \mathrm{~d}$ \\
\hline 5 & $0.46 \mathrm{a}$ & $0.94 \mathrm{~b}$ & $1.90 \mathrm{a}$ & $1.78 \mathrm{a}$ & $1.04 \mathrm{ab}$ & $0.82 \mathrm{bc}$ & $1.41 \mathrm{ab}$ & $1.29 \mathrm{a}$ & $1.93 \mathrm{ab}$ & $1.97 \mathrm{a}$ & $0.91 \mathrm{ab}$ & $0.64 \mathrm{be}$ & $1.97 \mathrm{a}$ & $1.32 \mathrm{ab}$ & $17.15 \mathrm{ab}$ \\
\hline Mean & 0.68 & 1.24 & 1.37 & 1.20 & 0.92 & 1.11 & 0.85 & 1.26 & 1.51 & 1.50 & 0.87 & 0.69 & 1.19 & 1.11 & 14.37 \\
\hline
\end{tabular}

1Means in the same column followed by the same letter do not differ statistically at the $5 \%$ probability level. 
C. plectostachyus Star was the most productive grass during the second year of experiment from February 1983 to February 1984, (table 4 ), although it was not significantly different $(\mathrm{P}=0.05)$ from $P$. maximum USDA PI 291047 and 259553 and cultivar Makueni. The production of Common Guinea and $D$. annulatus was similar and higher than that of C. nlemfuensis Star and C. dactylon Coastcross-1 $(\mathrm{P}=0.05)$, which did not persist at the end of the experiment.

Production during the second year was lower than that of the first year (tables 2 and 4), probably because of less rainfall in 1983, especially in June, July, and September, which are normally rainy (table 2). Apparently, irrigation was not sufficient to overcome the moisture deficit in the soil during dry spells.

Mean CP content of all grasses, except $D$. annulatus (pajón), varied from 11.19 to $14.05 \%$ (table 5). The CP content of $D$. annulatus (pajón) was only $9.73 \%$, indicating a lower nutritive value compared to that of improved species. The distribution of the CP content also varied according to the seasonal production of dry forage and it was lower during the periods of slow growth (fig. 1). Similar results have been found in the more humid northern coastal plains and humid mountains $(4,7,8,9)$ which have been explained by the dilution effect of yields in the presence of a uniform amount of $\mathrm{N}(11)$.

P. maximum USDA PI 259553 was highly productive in this experiment, as has been reported in more humid environments in Puerto Rico $(1,5,8)$. The same is applicable to cultivar Makueni $(7,8)$. These grasses are highly recommended for grazing trials to evaluate animal performance in all the different agricultural agroecosystems in Puerto Rico to compare results with those reported in the humid mountain region at Corozal (1).

\section{RESUMEN}

Producción de gramíneas tropicales pastadas en diferentes escosistemas agrícolas de Puerto Rico. III. Semiárido.

En un experimento que se realizó entre 1981 y 1984 en la Subestación Experimental de Lajas en la región semiárida de Puerto Rico se estudió la producción y persistencia de $\mathbf{8}$ gramíneas tropicales pastadas en parcelas pequeñas. Cynodon nlemfuensis estrella, C. dactylon coastcross-1, C. plectostachyus estrella, Panicum maximum USDAPI 291047, makueni y guinea común $P$. maximum USDA PI 259553 y el pasto natural Dichanthium annulatus pajón se pastaron a intervalos de 3 a 5 semanas a una altura de $15 \mathrm{~cm}$. por un grupo de 35 animales adultos por un período de 1 a 2 días. Las gramíneas más productivas durante el primer año del experimento fueron $P$. maximum makueni y USDA PI 291047, aunque no fueron diferentes $(P=0.05)$ de $P$. maximum USDA $P I 259553$, guinea común y $C$. plectostachyus estrella. La producción de $C$. nlemfuensis estrella fue similar a la de $D$. annulatus pajón, pero más alta que la de $C$. dactylon coastcross- 1 
TABLE 5.-Mean crude protein content" of dry forage on offer of tropical grasses under grazing in the semiarid region at Lajas, Puerto Rico, 1981 -1984

\begin{tabular}{|c|c|c|c|c|c|c|c|c|c|c|c|c|c|}
\hline \multirow[b]{2}{*}{$\begin{array}{l}\text { Grass } \\
\text { number }\end{array}$} & \multicolumn{12}{|c|}{ Grazing dates } & \multirow[b]{2}{*}{$\begin{array}{l}\text { Mean per } \\
\text { grazing }\end{array}$} \\
\hline & Jan. & Feb. & Mar. & Apr. & May & Jun. & Jul. & Aug. & Sep. & Oct. & Nov. & Dec. & \\
\hline \multicolumn{14}{|c|}{$\%$} \\
\hline 7 & 19.38 & 13.88 & 12.56 & 10.50 & 12.12 & 10.88 & 18.38 & 10.13 & 12.12 & 8.75 & 17.50 & 10.12 & 13.02 \\
\hline 2 & 16.25 & 15.38 & 10.50 & 14.25 & 14.12 & 8.12 & 17.38 & 10.63 & 11.13 & 6.75 & 17.25 & 9.25 & 12.58 \\
\hline 6 & 16.13 & 13:.63 & 11.50 & 12.12 & 11.75 & 9.75 & 17.38 & 12.00 & 11.13 & 10.00 & 16.13 & 9.25 & 12.81 \\
\hline 3 & 16.13 & 17.25 & 13.75 & 11.63 & 13.38 & 9.88 & 21.63 & 11.88 & 16.38 & 9.88 & 19.13 & 7.75 & 14.05 \\
\hline 4 & 16.00 & 14.25 & 11.63 & 10.50 & 11.25 & 9.50 & 17.38 & 13.25 & 9.63 & 8.88 & 13.38 & 10.87 & 12.21 \\
\hline 1 & 15.63 & 17.00 & 15.13 & 12.50 & 12.50 & 11.25 & 21.63 & 12.38 & 10.38 & 9.38 & 15.25 & 8.12 & 13.43 \\
\hline 5 & 13.38 & 13.25 & .9 .63 & 12.12 & 11.50 & 7.75 & 16.75 & 10.25 & 10.58 & 7.75 & 12.88 & 9.62 & 11.29 \\
\hline 8 & 13.38 & 11.25 & 8.25 & 6.88 & 10.50 & 6.63 & 15.63 & 8.99 & 6.50 & 5.38 & 15.00 & 8.37 & 9.73 \\
\hline Mean & 15.78 & 14.49 & 11.62 & 11.31 & 12.14 & 9.22 & 18.27 & 11.19 & 10.98 & 8.35 & 15.81 & 9.17 & \\
\hline
\end{tabular}

Mean of 4 replications for 2 years. 
$(P=.05)$. La producción media de todas las gramíneas disminuyó al comienzo del año durante los días cortos y frescos de febrero y marzo, 1983, $\sin$ que hubieran diferencias significativas $(P=0.05)$ entre especies y cultivares. Luego aumentaron hasta el comienzo de la estación lluviosa en mayo, pero fueron bajas hacia la época de lluvias máximas en octubre, debido probablemente a la pobre aireación y pisoteo en los suelos mal drenados y compactos. $C$. plectostachyus estrella fue la gramínea más productiva el segundo año del experimento en 1984, aunque no fue diferente $(P=0.05)$ de $P$. maxiumum USDA PI 291047 y 259553 y la cultivar makueni. La producción de guinea común y $D$. annulatus pajón fue similar y más alta que las de $C$. nlemfuensis estrella y $C$. dactylon coasteross-1 $(P=0.05)$, la cual no persistió al final del experimento. La producción durante el segundo año fue menor que durante el primero debido probablemente a una menor incidencia de las Iluvias en 1983, especialmente durante los meses de junio, julio y septiembre, los cualos son normalmente lluviosos. La media del contenido en proteína bruta fue mayor en las hierbas mejoradas que en la naturalizada $D$. annulatus pajón, y menor en todas las especies durante los períodos de máximo crecimiento. P. maximum USDA PI 259553 fue muy productiva en la región semiárida tal como se ha informado de otros ambientes más húmedos en Puerto Rico; lo mismo sucedió con la cultivar makueni.

\section{LITERATURE CITED}

1. Caro-Costas, R., 1980. Weight gains of cows fed on five grass pastures intensively managed in the humid hill region of Puerto Rico, J. Agric. Univ. P. R. 64 (1): 43-6.

2. Garefa-Molinary, 0., 1952. Grasslands and Grasses of Puerto Rico. Agric. Exp. Stn., Univ. P. R. Bull. 102.

3. Rivera, E. and J. Rodríguez, 1980. Forage yield of five grasses under intensive grazing management in the humid region of Puerto Rico, J. Agric. Univ. P. R. 64 (3): 259-63.

4. Soldevila, M., 1980. Effect of rotation length in pangola pastures upon the liveweight gain of growing Holstein heifers, J. Agric. Univ. P. R. 64 (2): 243-46.

5. Sotomayor-Ríos, A., A. Acosta-Matienzo and J. Vélez-Fortuño, 1971. Yield comparison and plant character correlations of 16 Panicum accessions, J. Agric. Univ. P. R. 55 (2): 174-83.

6. Tergas, L. E., J. Vélez-Santiago and D. Vera de Saldaña, 1988. Response of tropical grasses to potassium fertilization in the humid coastal region of Puerto Rico, $J$. Agric. Univ. P. R. 72 (1): 00.

7.,-- and,- 1988. Production of tropical grasses under grazing different agroecosystems in Puerto Rico. I. Humid mountain, J. Agric. Univ. P. R. 72 (1): 00.

8.,-- and,- 1988 . Production of tropical grasses under grazing in different agroecosystems in Puerto Rico. II. Humid northern coastal plains, J. Agric. Univ. P. R. 72 (1): 00 .

9. Vélez-Santiago, J., A. Sotomayor-Ríos, S. Torres-Rivera and A. V. Méndez-Cruz, 1982. Performance of six Cenchrus and four Panicum forage grasses under cutting management in the Lajas Valley, Puerto Rico, J. Agric. Univ. P. R. 66 (4): 268-77.

10. Vicente-Chandler, J., F. Abruña, R. Caro-Costas and S. Silva, 1983. Producción y utilización intensiva de las forrajeras en Puerto Rico, Esta. Exp. Agric, Unit. P. R. Bol. 271.

11. — , F. Abruña, R. Caro-Costas, J. Figarella, S. Silva and R. W. Pearson, 1974. Intensive Grassland Management in the Humid Tropics of Puerto Rico, Agric. Exp. Stn. Univ. P. R. Bull. 233. 